\title{
Navigating 2020 and beyond
}

As we usher in a new year of a new decade and ponder what the future will bring for the genetics field, we wish to reflect on some specific areas related to diversity, privacy and genome editing that require attention and vigilance from the community.

E xamining the field of genetics in 10 -year intervals, although somewhat an artificial construct, can be an interesting exercise. In the year 2000, we were galvanized by the sequencing of the human genome, and by 2010, the field had completely changed with the advent of next-generation sequencing. Looking back through our 2020 lens, we can appreciate the extreme rapidity with which the field has developed on a technological level. This advancement has been so fast that often the societal, legal and ethical consequences of these new techniques or capabilities have not been fully grappled with or resolved. Therefore, the field must carefully consider how genetics research affects various sectors, particularly with regard to how sequencing information is collected and used, and how new technologies are deployed and regulated.

The widespread availability of DNAsequencing data has afforded genetics research an unprecedented level of power to provide insight into diseases and complex traits. Cohorts in genome-wide association studies (GWAS) are now reaching more than 1 million individuals, owing to the establishment of large-scale biobanks, particularly the UK Biobank, and directto-consumer genetics testing companies, such as 23 andMe. However, these resources are heavily skewed toward individuals of European ancestry, and many of the findings of these analyses thus disproportionately benefit those populations specifically (for example, an analysis of polygenic risk scores; A. R. Martin et al. Nat. Genet. 51, 584-591; 2019). Therefore, increasing the diversity of genetic cohorts is imperative, as we have previously advocated (Nat. Genet. 51, 579; 2019). Doing so is important not just for GWAS studies but also for the study of rare diseases. In this issue, a Comment by Baynam et al. discusses the challenges involved in the diagnosis and treatment of rare genetic diseases in understudied populations, in this case those in Africa. Engagement with global communities must be thoughtfully planned, with sensitivity paid to local culture and practice. Another Comment by Wonkam and de Vries highlights this important point by discussing the need for Africa-specific guidelines to direct the return of results from genomics research in Africa. Our hope is that another 10 years will not be needed before we see appreciable change in the diversity of genetics research, but that a global perspective will be promoted and embraced at every opportunity moving forward.

As more and more people are having their genomes sequenced, concerns about security and privacy become increasingly important. The potential for data breaches or misuse of genetic information requires heighted awareness about the processes pertaining to the collection, storage and analysis of sequencing data. Specifically, in the obtaining of DNA samples, other ethical and legal considerations can come into play. For example, we are alarmed at the US Department of Homeland Security's plan in the immediate future to collect DNA samples from individuals in federal immigration custody at the United StatesMexico border and to store this information in the national crime database Combined DNA Index System (CODIS). This resource is used for forensic DNA analysis during the investigation of violent crimes, and including samples from individuals detained at the border has prompted privacy concerns. Equally troublingly, DNA evidence in criminal prosecutions is not foolproof, so having of thousands of these genetic samples could increase the risk of these individuals being falsely accused of a crime. What will programs such as these lead to in the future? In 2020 and beyond, all societal, ethical and legal consequences of mass DNA collection should be carefully weighed and monitored by independent, scientifically informed bodies.

More than a year has passed since the birth of two genome-edited babies was announced (D. Cyranoski and H. Ledford Nature 563, 607-608; 2018), and the debate over germline genome editing continues. Although we remain excited about what the next 10 years will hold in the realm of genome editing, and we encourage active dialogue in this area (Nat. Genet. 51, 365; 2019), we caution that any experimentation with human germline genome engineering via CRISPR or other methods must be carefully regulated. We must be sure that technology does not outpace our ability to responsibly handle the consequences of genome editing, and must include ethicists, legislators, disease advocates, researchers and patients in these complex discussions.

When we look ahead to 2030, or 2050 or 2100 , what will the genetics field look like? If 2010, 1990 and 1920 are anything to go by, we can expect that genetics and genomics will be practically unrecognizable 10, 20 or 100 years from now. Let's do all that what we can to drive the field forward scientifically while also ensuring that we steward it with maximum responsibility.

Published online: 7 January 2020 https://oi.org/10.1038/s41588-019-0570-0 